\title{
The Leon Wiltse Memorial Hostpital
}

The Leon Wiltse Memorial Hospital is a spine and joint specialized hospital located in Suwon, Korea. Dr. Choon Keun Park received his $\mathrm{MD}$ and $\mathrm{PhD}$ from the Catholic university of Korea medical school, and was a professor at the affiliated St. Vincent's hospital until founding the Wiltse Memorial Hospital in 2002. Dr. Park was one of the pioneers of minimally invasive spine surgery in Korea which he introduced after his 2 year fellowship at State University of New York (SUNY) in 1997.

At the time of the hospital's founding, specialized hospitals were yet an unfamiliar concept in Korea. The lack of a one stop center for the treatment of spine/joint diseases meant patients had to visit separate institutions for diagnosis, treatment, and rehabilitation. This resulted in redundancies and discontinuation of patient care. Dr. Park envisioned a comprehensive spine/ joint center focusing on the patient's disease which came to fruition through the Wiltse Memorial Hospital.

Dr. Leon Wiltse was one of the founders of the North American Spine Society (NASS). In this role, he was an important contributor to the establishment of modern spine surgery as an independent discipline. Dr. Park's mentor at SUNY was Dr. Yuan who trained under Dr. Wiltse. In the incubating stages of the hospital, Dr. Yuan's name was initially considered as the hospital's name. However, the current name was chosen upon Dr. Yuan's suggestion and Dr. Wiltse's blessings. The Wiltse Memorial Hospital cherishes the values exemplified by Dr. Wiltse's career - clinical excellence, academic rigor and medical education - and strives to demonstrate these ideals.

To realize these goals, the hospital has set its midterm goal to "serve as many patients as possible and provide better solutions for yet unsolved spine diseases."

Through high specialization and implementation of advanced healthcare practices the hospital has consistently grownover the past 13 years to become one of the most prominent specialty hospitals in Korea. The hospitals employs 22 experienced doctors who are also academically active having published 200 articles during the past decade. Outpatient visit has grown to 110000 yearly patients providing the broad foundation for our academic inquiry.

Korea is a rapidly aging society where healthcare for the elderly is an important topic. As spine and joint disease is a well-known degenerative disease, the Wiltse Memorial Hospital is facing higher expectations from the patients who present with increasingly complicated cases. To meet the various demands of the patients, we have expanded our spectrum of treatment. The hospi- tal was founded on the principles of minimally invasive spinal surgery but now practices a broad range of therapy, pain management and rehabilitation including but not limited to nerve blocks, nerve electrical stimulation, ligament strengthening, physical therapy and post-operative rehabilitation.

For patients who provide complicated, or non-responsive cases, novel therapies are employed. An example of such case is the "pain scrambler" which was adopted in 2013. Developed for patients who are unresponsive to known methods of treatment, it was approved by the ministry of health for public use in 2013. Since then, the hospital has practiced over 1000 cases and published various case reports to contribute in establishing indications and guidelines.

Dr. Park was selected as the 6th president of Korean Minimally Invasive Surgery Society in 2012, and currently serves as the president of the Spinal Therapeutic Technology related Link (STTL), and vice president of the Korean stem cell therapy society. STTL is sponsored by the ministry of trade industry and energy as a future national strategic industry. By following the lead of Dr. Park, the hospital and its staffare striving to achieve better care in innovative medicine while leading the discussions in the current field.

The hospital has focused on improving its standard of practice through extensive cooperation with government authorities. The government's policy to improve the current standard of medical practice has materialized by issuing several certifications to identify the leading clinics. This policy is in line with the Wiltse Memorial Hospital's principles and as such we have strived to meet every policy guideline. Our efforts have been recognized by patients, the medical field and government as we have been awarded all the recognized certificates issued by the government.

"As important as it is, we don't think patient satisfaction can be achieved by friendly service alone. The more important factor is the patient's confidence in the doctor and the hospital. We strive to achieve better results through improving established methods, upholding the principles of patient care, and developing innovative therapies." - Dr. Park

In 2011 the ministry of health recognized select clinics to be "government certified specialized hospitals" for their clinical aptitude for a 3 year term. Only 17 clinics received this certification out of which only 14 , including our hospital, received the certificate again in 2014. Also in 2011 the ministry of health selected a few "certified hospitals" which evaluated more than 400 infection, emergency, and patient care criteria. The Wiltse Memorial Hospital received both certificates in the first year of their 


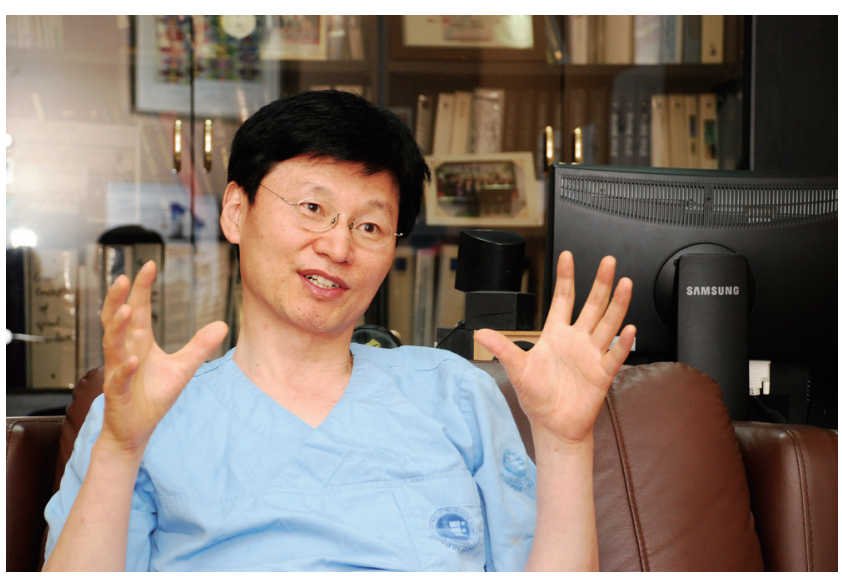

implementation, being one of the few institutions in Korea to do so.

Another important factor which differentiated the Wiltse Memorial Hospital from other Korean institutions is the lack of a care taker. Conventional Korean inpatient care requires a family member to take care of a patient while he is admitted. The nurses have a responsibility to administer drugs and injections but not to respond to everyday needs which is delegated to the patient's family or a hired care taker. The government sees this as at best an inefficiency and at worst a potential infection source. Our hospital agrees with this viewpoint and gladly volunteered to the pilot test of a "caretaker absent hospital" initiated in 2013 by the government where total patient care is initiated by the hospital in only 13 select clinics. Although this new system mandates the hospital to take the burden previously carried by the family members or hired caretakers, we judge the benefits to outweigh the substantial costs of hiring additional nursing staff, developing new protocol, and obtaining new equipment. Although the costs were substantial our efforts were well received as we have been recognized by the government, and more importantly the patients, as one of the more successful pilot cases.

The Wiltse Memorial Hospital has obtained measurable evidence of its higher standard of practice through the "government certified specialized hospital", "certified hospital" and "caretaker absent hospital" certifications. The reception of all three major government certification is achieved amongst very few institutions and has been recognized through the Prime minister's award. The hospital's excellence is also recognized by fellow institutions, many of which visit to learn our strengths.

Dr. Park is a pillar of the hospital and his enthusiasm is an in-

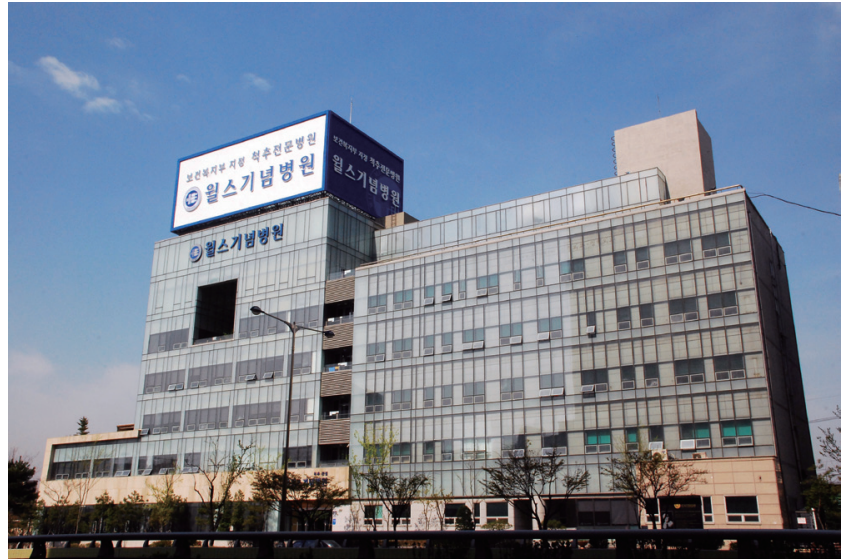

spiration for the staff. A regular day at Wiltse Memorial Hospital starts at 8:00AM with the conference of the day's operations and issues amongst the inpatients. There is a culture of academic tradition comparable to university affiliated hospitals. Dr. Park who himself was a professor at St. Vincent's - a catholic medical school affiliated hospital- implemented and nurtured the university hospital's high standards to the Wiltse Memorial Hospital.

"I understand the difficulties of maintaining high academic standards amongst our staff. They have a lot of tasks at hand even without research. However, research is a founding principle of this hospital and a mandatory criteria of a capable doctor. I hope our staff mature as a doctor during their time at our hospital" - Dr. Park

The Wiltse Memorial Hospital is expanding its expertise outside Korea. In 2007 the hospital was recognized as an Asia-Pacific minimally invasive spinal surgery training center. The same year we opened a spine clinic inside the Presidential medical center, Almaty, Kazakhstan by invitation which in 2014 additionally expanded a rehabilitation center sponsored by the world boxing federation. 20-30 doctors yearly visit the hospital for training from central Asia. We strive to uphold our principle value of education through such fellowships.

In the rapidly changing medical environment there are very few certainties. However, the Wiltse memorial hospital believes the future's challenges can be met by adhering to the principles which has served us well through the past 13 years; clinical excellence, academic rigor and medical education. We will continue to refine these concepts to ultimately better serve the suffering patients. 